\title{
Physical models of large number of asteroids from calibrated photometry sparse in time
}

\author{
M. Kaasalainen \\ Department of Mathematics and Statistics, Rolf Nevanlinna Institute, University of Helsinki, PO Box 4, 00014 Helsinki, Finland \\ e-mail: mjk@rni.helsinki.fi \\ Received 28 April 2004 / Accepted 15 June 2004

\begin{abstract}
I show that physical asteroid models can be constructed from unprecedentedly small sets of single calibrated photometric measurements sparse in time but well distributed in observing geometries. Sidereal periods, pole directions, and phase behaviour are solved with accuracy sufficient for the statistical analysis of a large collection of targets. Coarse estimates are obtained for the global shapes. This scheme makes it possible to carry out groundbased photometric surveys of thousands of asteroids, typically resulting in models within a few years. It may also be possible to model several asteroids using existing databases. In the future, GAIA photometry will provide another dataset for thousands of asteroids.
\end{abstract}

Key words. solar system: minor planets, asteroids - techniques: photometric - methods: numerical, observational

\section{Introduction}

When making disk-integrated photometric observations of an asteroid, the observer usually wants to obtain a good lightcurve, i.e., a long and dense time sequence well covering the target's rotational phases during the night. Often lightcurves are relative photometry, i.e., a sequence is only defined by its shape while its position in the absolute brightness scale is not accurately known. This is why the ordinary analysis mode of the method of lightcurve inversion (e.g., Kaasalainen et al. 2001, 2004, and references therein) relies much on relative photometry, and the absolute brightness values (when available) are used as constraints in the inverse problem, making sure that the result's light-scattering characteristics, in particular the solar phase curve, are consistent and realistic. Since absolute photometric values are usually less accurate than the relative ones, and the light-scattering model describing solar phase behaviour is not very well known, this is the only way of obtaining precise fits.

Measuring lightcurves is time-consuming, however. From the mathematical point of view, no lightcurves need be obtained. All one needs is a set of points at various observing geometries: technically their distances from each other in time is not a key factor when an underlying physical model is used. They just need to be calibrated in the absolute scale so that they can be directly compared with each other. For most asteroids very few or no lightcurves are available, but single brightness measurements at various ecliptic longitudes (and latitudes) can be made with accuracy sufficient for modelling the spin state and rough shape dimensions. This would also give improved value to even a few additional dense lightcurves already facilitating a preliminary global shape model.

\section{Inversion of sparse datasets}

The fast method of Kaasalainen et al. (2001) of optimizing a function series for the shape together with other parameters is directly usable also in this case: now we make just one "lightcurve" of all the observed brightness values, and find the best fit. Due to inaccuracies in both the light-scattering model and the observations, the fit cannot be expected to be as good as in the usual case of dense temporal sequences, and details in the model are certainly lost. However, we know that for suitable datasets with various observing geometries there is a distinct global minimum for period, pole, shape, and some scattering parameters, not lost even at high noise levels such as $5 \%$, which for most targets is roughly the level that can be expected to result from the combined error effects of observations and computational scattering models (Kaasalainen et al. 2001; Simonelli et al. 1996). The main question is how many points and observing geometries are needed for this.

The geometries are connected through orbital motion, so each case depends on its characteristic dynamics and the chosen observing epochs. I have simulated calibrated photometric observations in realistic long-time sequences to see how suitable sparse data are for this kind of inversion in practice. Real data sequences can be created by making sparse versions of "ordinary" well-calibrated lightcurve sequences. However, these sequences are far from optimal since particularly for main-belt asteroids (MBAs) the epochs are usually in a few 
tight groups. In reality epochs can be spread much better as lower solar elongations are possible since we do not have to cover any rotational phase interval in one go. Thus I also simulated photometric data in more appropriate sequences.

The empirical light-scattering model of Kaasalainen et al. (2001) is a practical choice in inversion as its three-parameter exponential-linear phase function is able to fit flexibly both the steep brightening effect at low solar phase angles and the linear decrease at larger ones. The full scattering model is obtained by combining the phase function with the Lommel-Seeliger and Lambert laws, with weight $c$ for the latter; this can be left as a free parameter, but the results are typically quite similar for $0<c<0.2$ (particularly for the expected model accuracy), so the fixed value $c=0.1$ was used as this further improved the convergence somewhat.

The first step in inversion is to find the correct period as this is the parameter that mostly dictates the model fit. The width $\Delta P$ of the scan interval is (typically slightly less than)

$\Delta P \approx \frac{1}{2} \frac{P^{2}}{T}$,

where $T$ is the full epoch range of the dataset, and $P$ is the period. Thus $\Delta P$ increases fast as a function of the trial period, and the number $N$ of scan intervals within the period range $\left[P_{0}, P_{1}\right]$ is

$N=\frac{2 T\left(P_{1}-P_{0}\right)}{P_{0} P_{1}}$

For the initial global period scan, one can also choose an initial subset with the shortest possible epoch range $T$ expected to suffice. Thus there are typically a few tens of thousands of initial period values, with some tens of shape and pole iterations for each check value. A personal computer can now do this in a few hours of CPU time. After the best period value(s) is/are found, it is a matter of minutes to find the best pole, shape, and refined period using a dense initial pole grid when starting from the best period(s). The procedure can readily be made fully automatic.

The effect of period is so strong that it is sufficient to scan the potential period range with a reduced set of initial pole values (and a sphere for initial shape) for each initial trial period. The ecliptic longitude $\lambda$ can typically be left to two initial values (separated by $90^{\circ}$ rather than $180^{\circ}$ due to the common $\lambda \pm$ $180^{\circ}$ ambiguity), and the latitude $\beta$ only requires initial values such as $\left(+45^{\circ}, 0^{\circ},-45^{\circ}\right)$. The fastest way is to scan the period range first by using the equatorial initial pole(s), and then switch to other latitudes if no clear best $\chi^{2}$ was found. It does not matter if the iteration for one trial period does not reach the best pole and shape: the procedure only needs some optimized shape and pole to be able to give a representative $\chi^{2}$-value for each interval. Within the period range there are various $\chi^{2}$-values, most fluctuating near the average level and many moderately good ones, but the best interval always appears to have a clearly lower $\chi^{2}$-level than the rest.

Figure 1 shows a part of the $\chi^{2}$-plot for the period scan of a calibrated photometry sequence of (433) Eros. The dataset consisted of only 59 points, each randomly picked from the Uppsala asteroid photometric catalogue (UAPC;

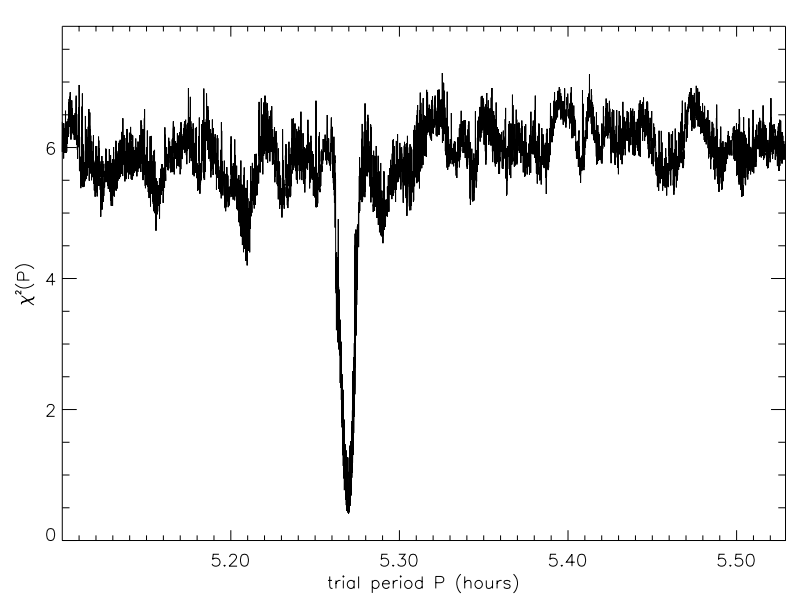

Fig. 1. Chi-square as function of initial period guess (and initial strongly offset pole) for sparse Eros data.

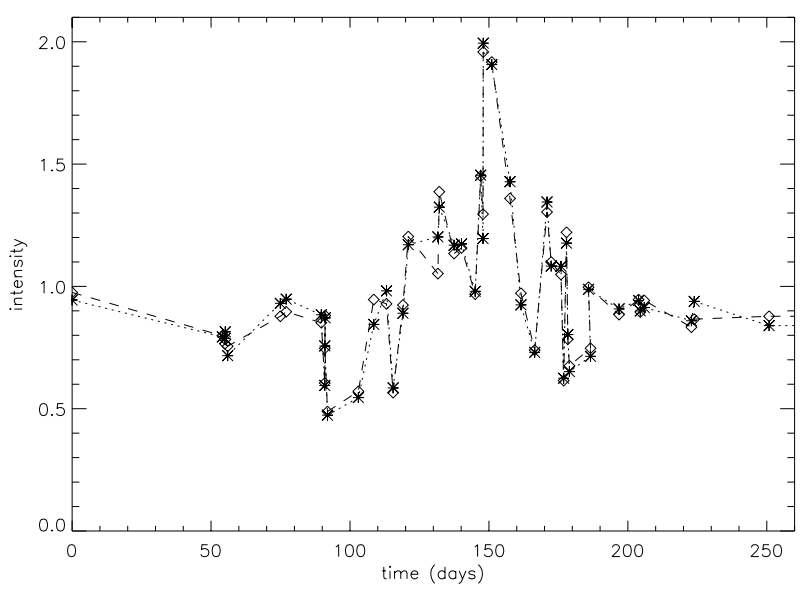

Fig. 2. Photometric observations (asterisks and dotted line) and the best model fit (diamonds and dashed line) for the Eros case (apparition 1974-75 shown here).

Lagerkvist 2001) lightcurves used in Kaasalainen et al. (2001) for Eros; just the two apparitions 1974-75 and 1981-82 were included here (one point from a lightcurve). The initial pole position for each trial $P$ was tens of degrees of arc away from the real pole, but the $\chi^{2}(P)$-signature is very strong, and the pole always converged fast to the right solution from all the periods around Eros' sidereal period $P=5.270255$ h. The best period and pole for this dataset, obtained with a dense pole grid for neighbouring best period scan slots, were $5.27025 \mathrm{~h}$ at $7^{\circ}$ of arc off the Kaasalainen et al. (2001) pole (although virtually as good a solution could be found $0.0004 \mathrm{~h}$ and $12^{\circ}$ off). As in ordinary photometric inversion, the fit reached the noise level, i.e., $0.05 \mathrm{mag}$ or $5 \%$ in relative intensity units in which the 1974-75 observations are plotted in Fig. 2. The observed points are marked with asterisks and connected with a dotted line, while the model points are diamonds with a dashed line. The lines are added only for visualization purposes: since the epochs are very sparse, they do not describe a lightcurve. The light-scattering parameters were within $\pm 15 \%$ of those reported in Kaasalainen et al. (2001), and the shape had the same elongated character. Similar results were obtained for other simulated NEA targets such as (25143) Itokawa or those in 
Kaasalainen et al. (2004): even two well-covered apparitions with less than a hundred points can be sufficient, so in the best cases a NEA model can be obtained in just a few years.

MBAs require slightly longer sequences, typically 4 apparitions. Figure 3 shows the period scan for a Gaspra-shaped target in (4) Vesta's orbit, and the best fit is shown in Fig. 4; the data were again fitted down to the noise level $0.04 \mathrm{mag}$. The sequence was made by using only elongations over $60^{\circ}$ (and the complete solar phase angle range $2^{\circ}-28^{\circ}$ ) making the target well observable at all epochs. The timeline was 4.4 years, consisting of 4 apparitions and 129 random epochs with intervals from one day to two weeks. It is important to gather such sequences with varying intervals to avoid sampling resonances. Despite the noise, the best pole was only $2^{\circ}$ away from the correct one, with estimated error $\pm 10^{\circ}$. Since the target moves close to the plane of the ecliptic, the pole solution also has the $\lambda \pm 180^{\circ}$-ambiguity. The period strongly peaks at the correct value of $9 \mathrm{~h}$. This is a good example of how physical modelling allows accurate period analysis without aliasing effects even for datasets for which the sampling density is considerably lower than that typically needed for model-independent time series methods. The range of trial periods was from 2 to $20 \mathrm{~h}$ in this and the Eros case (not shown in $\chi^{2}$-plots for clarity as there were no rival period solutions far from the main peak).

The example above as well as various other sequences and orbits were simulated using realistic shape models and typical values of the Hapke model or exponential-linear parameters for light scattering; in most cases the parameters for the latter were determined to about $\pm 10 \%$. As discussed in Kaasalainen et al. (2001), the two models produce similar results both in direct and inverse problem, but the former is considerably easier to use in inversion particularly in the linear $\alpha \gtrsim 15^{\circ}$-region of the solar phase function. The pole directions were $2^{\circ}$ to $15^{\circ}$ of arc off the correct ones, with error estimates $\pm 5^{\circ}- \pm 15^{\circ}$ of arc based on $\chi^{2}$-distribution (error mostly correlating with accuracy), and the period accuracy (and error) was typically a tenth part of the scan interval Eq. (1). The shape solutions are naturally only coarse representations of the original shapes, but their dimension ratios are within some $\pm 15 \%$ of the correct ones, sufficient for classification (note that the concept of dimension ratio is not well defined for arbitrary bodies: one choice is a mean value of ratios based on the largest extents and the best-approximating ellipsoid). The average rms fit deviation level for incorrect spin parameters typically varied roughly between 0.06-0.10 mag in our simulations, so it indeed seems that the combined observational and light-scattering model inaccuracy should not much exceed 0.05 mag if we want the correct solution to stand out. It should also be added that these examples were of "ordinary" asteroids; potentially problematic cases such as very fast/slow rotators, tumblers, binaries, or several outlier points will be studied in a longer future paper.

\section{GAIA photometry}

During its five-year mission, the GAIA satellite observatory is expected to obtain photometric measurements of thousands of asteroids, typically less than one hundred points for each. The expected epoch sequence for (4) Vesta (F. Mignard,

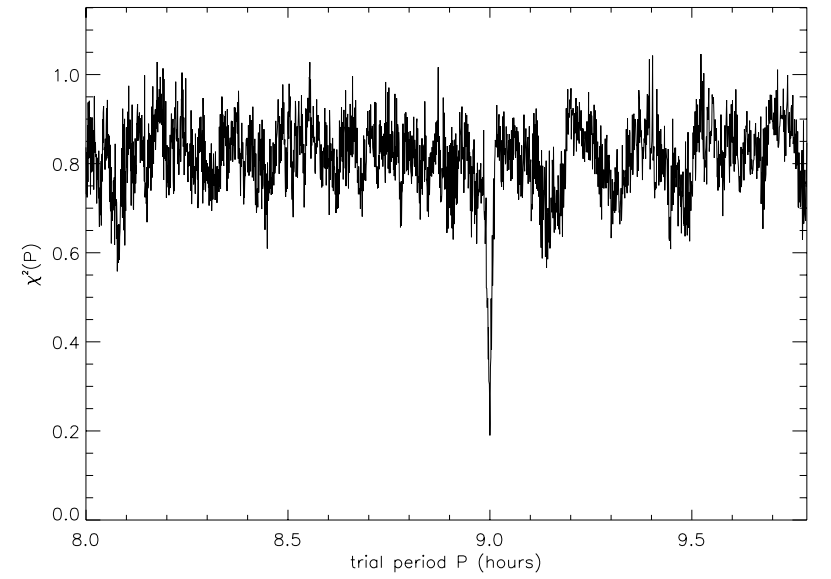

Fig. 3. Chi-square as function of initial period guess (and initial strongly offset pole) for simulated sparse MBA data.

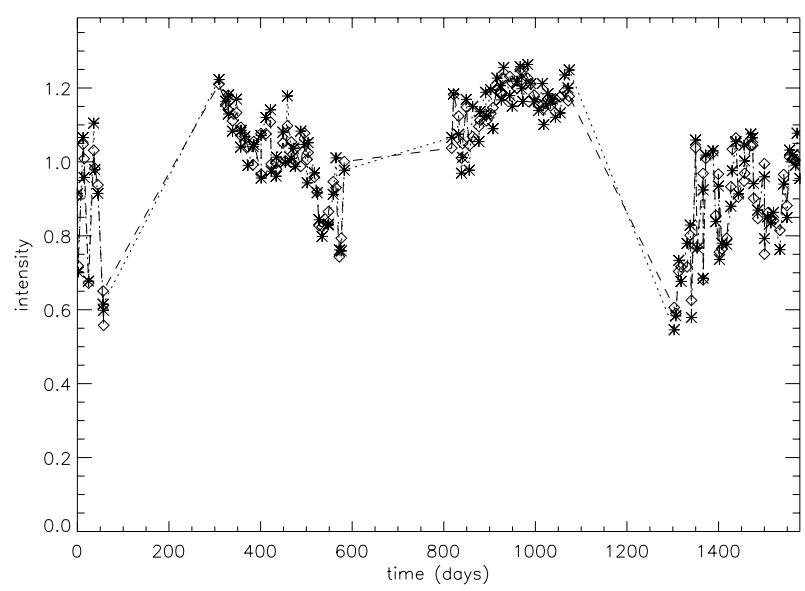

Fig. 4. Simulated observations (asterisks and dotted line) and the best model fit (diamonds and dashed line) for the MBA-example.

K. Muinonen, private communication) spans the same timeline as the MBA-example above, and an inversion example is shown in Figs. 5 and 6. The target properties are the same as in the MBA-example, and 0.03 mag noise was added to the data. The time sequence covers the four-year range quite evenly with 69 epochs. The gaps between the four apparitions are not very wide as the lowest solar elongations are $42^{\circ}$. For this dataset, the solar phase angle remained between $15^{\circ}$ and $28^{\circ}$, typical for GAIA observations. Now the best-fit solution was $12^{\circ}$ away from the correct pole, and the rms deviation of the fit was $3 \%$. Again the best period stood out very clearly.

As discussed in Kaasalainen \& Tanga (2004), the photocentre offset of an asteroid is non-negligible for many targets, so models obtained in the above manner will also improve the accuracy of barycentre determination with GAIA. Moreover, GAIA's astro- and photometric data can be combined in a full inverse problem in which both the orbit parameters and the shape/spin model are simultaneously improved (Kaasalainen et al., in preparation). 


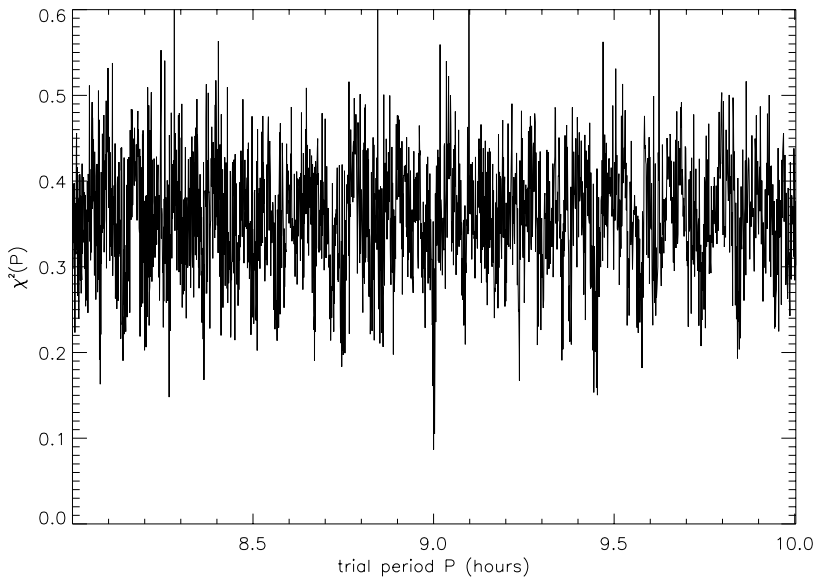

Fig. 5. Chi-square as function of initial period guess (and initial strongly offset pole) for simulated sparse GAIA data.

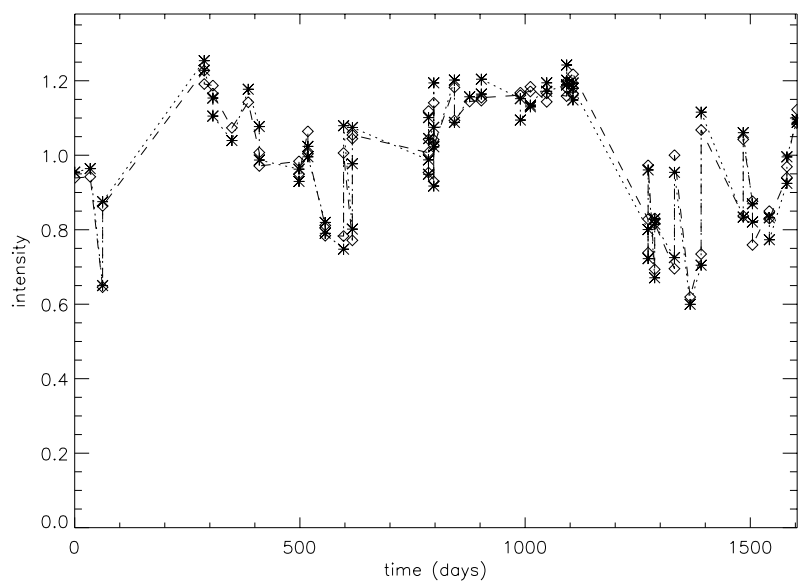

Fig. 6. Simulated observations (asterisks and dotted line) and the best model fit (diamonds and dashed line) for GAIA-case.

\section{Conclusions}

The information content of sparse photometric data is surprisingly high. As a rule of thumb, about one hundred calibrated data points within four to five years should well suffice for obtaining the sidereal period and pole direction of an MBA, and for getting a rough idea of its shape and solar phase behaviour. NEA models may be obtained even faster. If the accuracy of the data points is at least about $0.05 \mathrm{mag}$, the procedure finds the global minimum and converges very robustly.

There may already be asteroids for which suitable calibrated measurements exist in various databases (e.g.,
Astrovirtel, Hipparcos, or campaigns such as Lagerkvist et al. 1992) that should be mined for the purpose. It should now also be possible to initiate photometric surveys that use particularly wide-field telescopes in systematic sky-sweeping mode to scan asteroids for several years. It would also be very useful to improve the photometric accuracy of astrometric observation programs. Such surveys would result in several thousands of asteroid models in the near future, and they would also be important backup and comparison/complementary data sources for GAIA photometry. Investigations of optimal groundbased epoch sequences and survey strategies as well as simulations and analysis of GAIA asteroid photometry will be presented in forthcoming papers.

A large database of asteroid spin states and basic shapes is necessary for the studies of asteroids' dynamical and physical evolution particularly in the light of recent results (e.g., Vokrouhlický et al. 2003; Nesvorný et al. 2002; La Spina et al. 2004). It is possible to compile such a database with both groundbased and space observations without having to observe traditional lightcurves at all. This approach would be roughly two orders of magnitude more time-efficient than the current one.

Acknowledgements. It is my pleasure to thank A. Harris, P. Pravec, S. Lowry, B. Warner, and J. Torppa for valuable comments and discussions, and F. Mignard and K. Muinonen for a sample GAIA epoch sequence.

\section{References}

Kaasalainen, M., Torppa, J., \& Muinonen, K. 2001, Icarus, 153, 37

Kaasalainen, M., Pravec, P., Krugly, Yu. N., et al. 2004, Icarus, 167, 178

Kaasalainen, M., \& Tanga, P. 2004, A\&A, 416, 367

Lagerkvist, C.-I., Magnusson, P., Williams, I. P., et al. 1992, A\&AS, 94,43

Lagerkvist, C.-I., Piironen, J., \& Erikson, A. 2001, UAPC, Fifth update (Uppsala: Uppsala University Press)

La Spina, A., Paolicchi, P., Kryszczynska, A., \& Pravec, P. 2004, Nature, 428, 400

Nesvorný, D., Bottke, W. F., Dones, L., \& Levison, H. F. 2002, Nature, 417,720

Simonelli, D. P., Veverka, J., Thomas, P. C., Helfenstein, P., \& Carcich, B. T. 1996, Icarus, 120, 38

Vokrouhlický, D., Nesvorný, D., \& Bottke, W. F. 2003, Nature, 425, 147 